\title{
FATORES ASSOCIADOS A NÃO ADESÃO AO TRATAMENTO DA HIPERTENSÃO ARTERIAL SISTÊMICA: UMA REVISÃO INTEGRATIVA
}

\author{
FACTORS ASSOCIATED WITH NON-ADHERENCE TO TREATMENT OF SYSTEMIC \\ HYPERTENSION: AN INTEGRATIVE REVIEW
}

\author{
Leidiane Mirlla e Oliveira Mendes ${ }^{1}$ \\ Jacira da Silva Torres e Barros ${ }^{1}$ \\ Nancy Nay Leite de Araújo Loiola Batista ${ }^{2}$ \\ Joselma Maria Oliveira Silva ${ }^{3}$
}

\begin{abstract}
Resumo: O controle inadequado da pressão arterial pode estar relacionado à falta de adesão do paciente hipertenso ao tratamento indicado. Objetivo: Sintetizar o conhecimento produzido e publicado na literatura nacional sobre os fatores associados à não adesão ao tratamento da hipertensão arterial no período de 2006 a 2012. 0 presente estudo trata-se de uma revisão integrativa da literatura. O levantamento bibliográfico ocorreu mediante a consulta na base de dados online da Biblioteca virtual em Saúde. Critérios de inclusão: artigos nacionais, textos completos, que abordassem a temática e os publicados entre 2006 a 2012. Critérios de exclusão: que não estavam relacionados à temática do estudo e que estavam fora do período determinado. Foram considerados 11 artigos. 0 ano de 2008 e 2010 concentrou o maior número de publicações com 28\% cada. Observaram-se, nos artigos, as abordagens metodológicas qualitativa com $27 \%$ e quantitativa com $73 \%$. A região Sudeste se destaca com $37 \%$ das publicações. Local de realização das pesquisas, 91\% dos artigos foram realizados em Unidades Básicas de Saúde. 19\% dos artigos foram publicados na Revista Rene. Procedeu-se uma leitura analítica que possibilitou a formulação da categoria: Fatores que influenciam a não adesão ao tratamento anti-hipertensivo. O estudo realizado permitiu sintetizar a produção de conhecimento nacional acerca dos fatores que influenciam a não adesão ao tratamento da hipertensão. A compreensão dos reais motivos da não adesão ao tratamento da hipertensão se torna importante no que se refere à criação de projetos e ações de saúde pública voltada para esse agravo.
\end{abstract}

Palavras-chave: enfermagem; hipertensão; tratamento; fatores.

Abstract: Inadequate control of blood pressure may be related to lack of adherence of hypertensive patients to their treatment. Objective: To summarize the information produced and published in the literature on the factors associated with non-adherence to hypertension treatment in the period 2006 to 2012. This study deals with an integrative literature review. The bibliography was compiled by consulting the online database of the Virtual Library in Health. Inclusion criteria: national papers, full texts that dealt with the subject and were published between 20062012. Exclusion criteria: were not related to the topic of the study and were outside the specified period. Eleven articles were considered. The years 2008 and 2010 had the largest number of publications with $28 \%$ each. The methodological approaches of the articles were $27 \%$ qualitative and $73 \%$ quantitative. The Southeast region stands out with $37 \%$ of the publications; $91 \%$ of the reseach for the articles was conducted in Primary Health Units and $19 \%$ of the articles were published in the Revista Rene. Reading of the articles enabled the analytical formulation of the category: Factors influencing non-adherence to antihypertensive treatment. The study has allowed us to synthesize the production of national knowledge about the factors influencing non-adherence to treatment of hypertension. Understanding the real reasons for non-adherence to treatment of hypertension becomes important for the creation of projects and public health actions directed to this condition.

Keywords: nursing; hypertension; treatment; factors.

\footnotetext{
1 Graduanda do Curso de Enfermagem - Faculdade Santo Agostinho - FSA. E-mails: leidianemirlla@hotmil.com; jaciratb@hotmail.com.

2 Mestre em Saúde da Família - Universidade Federal do Piauí - UFPI e Professora da FSA. E-mail: nancyloiola@uol.com.br.

${ }^{3}$ Graduada em Fisioterapia - FSA e Especialista em Docência do Ensino Superior - Centro de Ensino Unificado de Teresina - CEUT. E-mail: joselma_01@hotmail.com.

Revista Univap - revista.univap.br

São José dos Campos-SP-Brasil, v. 20, n. 35, jul.2014. ISSN 2237-1753
} 


\section{INTRODUÇÃO}

As doenças cardiovasculares (DCV) representam a primeira causa de morte no Brasil. Apesar da tendência de redução dos riscos de mortalidade por DCV no País e no mundo, algumas projeções indicam o aumento de sua importância relativa em países de baixa e média renda. A maior longevidade, associada ao possível aumento da incidência das DCV por adoção dos modos de vida com maior exposição a fatores de risco, são consideradas as principais razões desse incremento. Como fatores de risco, estão o tabagismo e inatividade física, além de dieta rica em gorduras saturadas, com consequente aumento dos níveis de colesterol e hipertensão (ISHITANI et al., 2006).

As doenças do aparelho circulatório foram responsáveis por mais de 800 mil internações processadas pelo Sistema Único de Saúde (SUS), em 2006, com um custo aproximado, de 1 bilhão e 300 milhões de Reais e se mantêm como principal causa agrupada de mortes no Brasil. A Região Sul apresenta a maior taxa de internações por doenças cardiovascular, os custos médios de internação mais elevados, e as maiores taxas brutas de mortalidade do país (SANTA-HELENA; NEMES; ELUF NETO, 2010).

No Brasil, um fato que agrava esse quadro é que, aproximadamente, um terço dos óbitos por doenças do aparelho circulatório ocorrem precocemente, em adultos na faixa etária de 35 a 64 anos. Nessa faixa etária, as principais causas de óbito são por isquêmicas do coração, as doenças cerebrovasculares e as doenças hipertensivas. Ressalta-se que essas causas são, em grande parte, evitáveis, diante da probabilidade de diminuição da ocorrência dessas mortes, se houver assistência ou prevenção oportuna (ISHITANI et al., 2006).

A hipertensão arterial é um problema crônico comum. Sua prevalência é alta e aumenta em faixas etárias maiores. Estudos epidemiológicos brasileiros estimam prevalência de $40 \%$ a $50 \%$ da população adulta com mais de 40 anos, a partir da medida casual da pressão. Mesmo sendo assintomática, a hipertensão arterial é responsável por complicações cardiovasculares, encefálicas, coronarianas, renais e vasculares periféricas. Estima-se que $40 \%$ dos acidentes vasculares encefálicos, e em torno de $25 \%$ dos infartos ocorridos em pacientes hipertensos, poderiam ser prevenidos com terapia anti-hipertensiva adequada. No entanto, parcela importante da população adulta com hipertensão não sabe que é hipertensa; e muitos dos que sabem não estão sendo adequadamente tratados (TOSCANO, 2004).

As complicações da hipertensão arterial, em muitos casos, levam o paciente a requerer cuidados médicos de alto custo, exigindo uso constante de medicamentos, exames complementares periódicos e procedimentos como diálise e, até mesmo, transplante. No Brasil, as doenças cardiocirculatórias são uma das principais causas de internações hospitalares e, reconhecidamente, envolvem custos elevados (COSTA et al., 2007).

Mesmo com o grande avanço científico e tecnológico no manejo da hipertensão arterial ocorrido nos últimos anos, uma das grandes dificuldades atuais refere-se à adesão dos pacientes aos tratamentos instituídos, ou seja, até que ponto o paciente segue as recomendações dadas pelo médico ou outro profissional de saúde para o controle do seu problema. Considera-se adesão a um tratamento o grau de coincidência entre a prescrição médica, o que inclui as orientações não farmacológicas e o comportamento adotado, 
concretamente, pelo paciente. No caso da Hipertensão Arterial Sistêmica (HAS), envolve a extensão em que o comportamento do indivíduo (em termos de uso efetivo do medicamento, realização de mudanças no estilo de vida e comparecimento às consultas médicas) coincide com o conselho médico. Assim, o controle inadequado da pressão arterial pode estar relacionado à falta de adesão do paciente hipertenso ao tratamento indicado (MANFROI; OLIVEIRA, 2006).

Inúmeros estudos têm apontado a enorme dificuldade vivida pelas pessoas com hipertensão para persistirem seguindo as recomendações médicas, com expressiva frequência de abandono do tratamento. Estima-se que cerca de dois terços dos pacientes com Hipertensão Arterial (HA) não têm seus níveis pressóricos adequados, devido, em grande parte, ao seguimento incorreto do tratamento medicamentoso. Estudos internacionais e nacionais mostram grande variação nas taxas de adesão e abandono observadas. Essa variação deve-se a vários fatores, entre eles o método de medida utilizado, o ponto de corte adotado para a definição de adesão e a seleção da amostra estudada. É importante, também, reconhecer que a assistência a pessoas com doenças crônicas, como a HA, o diabetes e outras, requer considerar a complexidade do cuidado (e do autocuidado) em condições de cronicidade. Destaca-se, nesse reconhecimento, a crítica à abordagem estritamente técnica da adesão do paciente e à restrita consideração das dificuldades vividas em seu cotidiano (DUARTE et al., 2010).

No cuidado a esta clientela, sobressai o enfermeiro como o profissional responsável por tal cuidado ao acompanhá-la sistematicamente. Desta maneira, ele poderá colaborar na minimização das barreiras ao tratamento anti-hipertensivo, por meio de orientações, incentivo, acolhimento, escuta qualificada, uso dos recursos disponíveis no serviço para complementar a assistência, valorização de suas dificuldades, medos e objeções ao tratamento (GUEDES et al., 2011).

Diante dessa realidade, a equipe de saúde que trabalha diretamente com os pacientes portadores de HAS deve estar apta a fornecer orientações e assistência adequada referente ao tratamento da HAS, para que possibilite evitar complicações e o abandono do tratamento. Assim, espera-se que os resultados desta pesquisa contribuam para a elaboração de medidas que possibilitem o aumento da adesão ao tratamento da HAS, reduzindo os gastos públicos com internações e morbimortalidade decorrentes das complicações da hipertensão arterial.

O presente artigo tem como objetivo sintetizar o conhecimento produzido e publicado na literatura nacional sobre os fatores associados à não-adesão ao tratamento antihipertensivo, no período de 2006 à 2012.

\section{METODOLOGIA}

Trata-se de uma revisão integrativa da literatura, que busca responder à seguinte questão norteadora: Qual a produção técnica científica sobre os fatores associados a não adesão ao tratamento da hipertensão arterial?

Esse tipo de abordagem metodológica é considerado o mais amplo entre as revisões, Revista Univap - revista.univap.br

São José dos Campos-SP-Brasil, v. 20, n. 35, jul.2014. ISSN 2237-1753 
pois permite incluir literatura teórica e empírica, bem como estudo com diferentes abordagens metodológicas. A revisão integrativa tem como principal finalidade reunir e sintetizar os estudos realizados sobre um determinado assunto, construindo uma conclusão a partir dos resultados evidenciados em cada estudo (POMPEO; ROSSI; GALVÃO, 2009; SOUZA; SILVA; CARVALHO, 2010).

De acordo com Pompeo, Rossi e Galvão (2009), esse é um método que permite gerar uma fonte de conhecimento atual sobre o problema e determinar se o conhecimento é válido para ser transferido à prática. Assim, a construção da revisão integrativa deve seguir padrões de rigor metodológico, os quais possibilitam ao leitor identificar as características dos estudos analisados.

O estudo proposto decorreu em várias etapas, essas são partes integrantes de uma revisão integrativa, a conhecer: escolha do tema, formulação do problema e objetivo da revisão integrativa, estabelecimento de critérios de inclusão e exclusão dos artigos, levantamento bibliográfico (seleção da amostra), definição das informações a serem extraídas dos artigos selecionados, leitura e interpretação para a análise do material que foi finalizado com a produção textual.

O levantamento bibliográfico ocorreu mediante a consulta na base de dados online da Biblioteca virtual em Saúde (BVS), selecionou-se, no campo de preenchimento, a opção (todas as fontes), por possibilitar a inclusão de um número significativo de trabalhos, que objetivou minimizar possíveis vieses no processo de elaboração da revisão integrativa.

O estudo foi realizado utilizando-se a combinação dos descritores hipertensão, tratamento e não adesão. Os critérios de inclusão dos textos foram: artigos nacionais, textos completos que fossem disponíveis e que abordassem a temática hipertensão e fatores associados à não adesão ao tratamento, publicados entre 2006 a 2012. Foram excluídos os artigos que não estavam relacionados à temática do estudo, os que estavam fora do período determinado, bem como as repetições. A seleção do material ocorreu nos meses de setembro a novembro de 2012.

Utilizou-se, na procura dos artigos, as palavras hipertensão, tratamento, adesão e foram encontrados 102 artigos científicos na temática indicada. Durante a pesquisa, houve referências que não respondiam ao interesse temático, outras que não disponibilizavam o texto completo e, também, artigos que não contemplavam o período do estudo. Portanto, os que abrangiam o enfoque da pesquisa com seus critérios de inclusão, e foram considerados, para a concretização desse estudo, 11 artigos (Figura 1). 


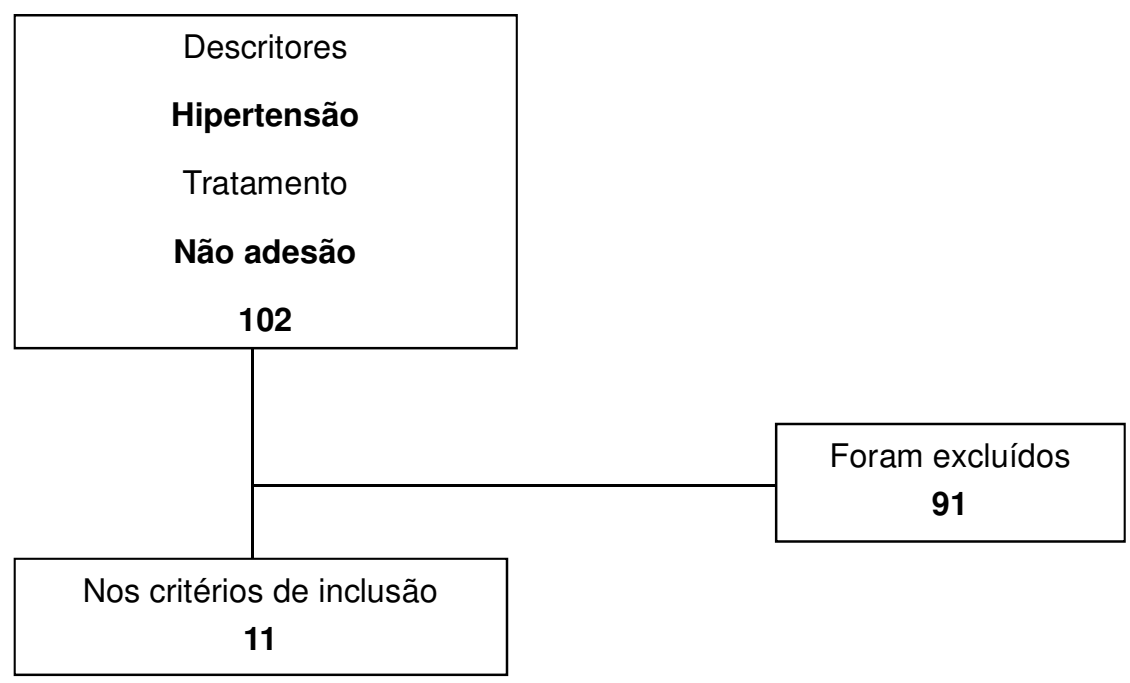

Figura 1 - Fluxograma da seleção dos artigos do estudo.

Fonte: Própria do autor.

Os artigos foram lidos e analisados por intermédio das seguintes variáveis: Ano de publicação, abordagem metodológica, região geográfica na qual o estudo foi realizado, local de realização da pesquisa, periódico de publicação e foco principal. Para melhor ordenação dos dados, foi elaborado um formulário (APÊNDICE A), que facilitou a análise dos dados e a busca de conteúdo acerca dos fatores associados à não adesão ao tratamento da hipertensão.

\section{RESULTADOS E DISCUSSÃO}

Após a seleção e análise dos artigos, foram estabelecidas algumas variáveis relevantes para apreciação das produções cientificas da temática pesquisada, conforme se observa na Tabela 1. 
Tabela 1 - Distribuição das produções científicas segundo o período de publicação, abordagem metodológica, região geográfica, local de realização do estudo e periódico de publicação $\left(\mathrm{n}^{\circ}=11\right)$. Teresina-PI, 2012

\begin{tabular}{|c|c|c|}
\hline Variáveis & $\begin{array}{l}\text { Número de } \\
\text { publicações }\end{array}$ & $(\%)$ \\
\hline \multicolumn{3}{|l|}{ Período } \\
\hline 2006 & 1 & 9 \\
\hline 2007 & 1 & 9 \\
\hline 2008 & 3 & 28 \\
\hline 2009 & 1 & 9 \\
\hline 2010 & 3 & 28 \\
\hline 2011 & 2 & 19 \\
\hline 2012 & --- & 0 \\
\hline \multicolumn{3}{|l|}{ Abordagem Metodológica } \\
\hline Quantitativo & 8 & 73 \\
\hline Qualitativo & 3 & 27 \\
\hline \multicolumn{3}{|l|}{ Região Geográfica } \\
\hline Sudeste & 4 & 37 \\
\hline Nordeste & 3 & 27 \\
\hline Centro-Oeste & 3 & 27 \\
\hline Sul & 1 & 9 \\
\hline Norte & --- & 0 \\
\hline \multicolumn{3}{|l|}{ Local de Realização } \\
\hline Unidade Básica de Saúde & 10 & 91 \\
\hline Ambulatório/Centro de Saúde & 1 & 9 \\
\hline \multicolumn{3}{|l|}{ Periódicos } \\
\hline Rev. Rene & 2 & 19 \\
\hline Rev. Acta Paulista de Enfermagem & 1 & 9 \\
\hline Rev. Brasileira de Enfermagem & 1 & 9 \\
\hline Rev. Cogitare Enfermagem & 1 & 9 \\
\hline Rev. Mineira de Enfermagem & 1 & 9 \\
\hline Rev. Caderno de Saúde Pública & 1 & 9 \\
\hline Rev. Ciências Saúde Coletiva & 1 & 9 \\
\hline Rev. Ciências Farmacêuticas Básica e Aplicada & 1 & 9 \\
\hline Rev. Temas Livres & 1 & 9 \\
\hline Rev. Redalyc & 1 & 9 \\
\hline
\end{tabular}

Fonte: BVS.

Verificou-se que os anos 2008 e 2010 concentraram o maior número de publicações, com 28\% cada, seguido do ano de 2011, com 19\%; já, os anos de 2006, 2007 e 2009 concentraram o menor número de publicação, com 9\% cada. Observou-se, nos artigos, a aplicação de tipos diferentes de abordagem metodológica, como qualitativa, com $27 \%$, e a abordagem quantitativa, mais frequente, com $73 \%$, que, em sua maioria, abordavam variáveis sobre os aspectos sócios demográficos que influenciam na adesão ao tratamento antihipertensivo e fatores para não adesão.

Segundo Teixeira (2002), a pesquisa quantitativa utiliza a descrição matemática como linguagem para descrever as características de um fenômeno. A estatística faz a relação entre a teoria apresentada nos livros e os dados no ambiente da pesquisa. 
Quanto à região geográfica, sobressaiu-se a região Sudeste, com 37\% das publicações, isso se justifica pela maior concentração de Universidades, como, também, hospitais escolas nessa região; em seguida, vêm as regiões Nordeste e Centro-Oeste, com 27\%, cada; e o Sul, com $9 \%$. Na região Norte, não foi encontrado nenhum estudo.

Em relação ao local de realização da pesquisa, observou-se que $91 \%$ dos artigos $(n=10)$ foram realizados em Unidades Básicas de Saúde (na Estratégia Saúde da Família ESF). Esse local se configura em um cenário ideal para o tipo de estudo proposto pelos pesquisadores, pois fornece uma maior fonte de dados referente à população de hipertensos, em decorrência do programa assistencial ao HIPERDIA (Hipertensos e Diabéticos). Já, o outro local citado foi o Ambulatório (Centro de Saúde), que representou apenas 9\%.

O HIPERDIA é um plano de reorganização da atenção à hipertensão arterial, que permite cadastrar e acompanhar os hipertensos em todas as unidades ambulatoriais do SUS, e que garante o recebimento dos medicamentos prescritos. Além disso, é uma ferramenta útil que gera informações para os gestores da saúde e Ministério da Saúde a respeito do perfil epidemiológico da população, a fim de propor estratégias, visando à melhoria da qualidade de vida dessas pessoas (LOPES; FERREIRA; MARIANO, 2009).

Quanto aos periódicos nos quais os artigos foram publicados, encontraram-se poucas variações nas distribuições desses artigos, destacando-se a Revista Rene, com 19\% ( $n=2)$, os demais artigos tiveram uma distribuição semelhante, com $9 \%(n=1)$ cada, totalizando $81 \%$ das revistas analisadas.

Após a análise dos periódicos, constatou-se que $73 \%$ dos artigos selecionados para o estudo foram elaborados por enfermeiros e os $27 \%$ restantes foram produzidos por médicos e farmacêuticos. Observou-se, também, que 55\% $(n=6)$ dos artigos analisados encontravamse em revistas especializadas (Revista de Enfermagem) e $45 \%(n=5)$ dos artigos acharam-se em periódicos diversificados.

Ao se analisar esses periódicos, percebeu-se que a existência de revista específica de enfermagem contribui com a divulgação do conhecimento produzido na área e, também, demonstra a busca permanente dos enfermeiros na atualização do conhecimento baseado em evidências, na sua prática profissional.

Os artigos selecionados fazem referência aos fatores que contribuem à não adesão ao tratamento anti-hipertensivo, às dificuldades da adesão ao tratamento relatado pelos indivíduos hipertensos, motivo do abandono ao seguimento no cuidado ao hipertenso e fatores sócio demográficos, os quais interferem na adesão ao tratamento da hipertensão. Com o material dos artigos selecionados e a delimitação do foco principal (Quadro 1), procedeu-se uma nova leitura analítica, que possibilitou reunir os fatores associados à não-adesão ao tratamento da hipertensão arterial descritos na literatura atual. Desse modo, suscitou do estudo uma categoria a ser estudada. Essa categoria é apresentada a seguir. 


\section{Quadro 1 - Distribuição dos artigos publicados no período de 2006 a 2011 segundo o foco} principal ( $\mathrm{n}=11)$. Teresina-PI, 2012

\begin{tabular}{|c|l|}
\hline $\mathbf{N}^{\circ}$ & \multicolumn{1}{|c|}{ FOCO } \\
\hline $\mathbf{1}$ & Motivo do abandono do seguimento médico no cuidado a portadores de hipertensão \\
\hline $\mathbf{2}$ & $\begin{array}{l}\text { Fatores dificultadores que levam os portadores de hipertensão à não aderência ao } \\
\text { tratamento anti-hipertensivo }\end{array}$ \\
\hline $\mathbf{3}$ & Dificuldades relatadas por indivíduos hipertensos na adesão ao tratamento \\
\hline $\mathbf{4}$ & Controle hipertensão arterial e fatores associados \\
\hline $\mathbf{5}$ & Barreiras ao tratamento da hipertensão arterial \\
\hline $\mathbf{6}$ & Fatores relacionados à não-adesão ao tratamento da hipertensão \\
\hline $\mathbf{7}$ & Fatores para não adesão ao programa de controle da hipertensão arterial \\
\hline $\mathbf{8}$ & Fatores que contribuem à não-adesão ao tratamento com anti-hipertensivos \\
\hline $\mathbf{9}$ & Adesão às medidas de controle da Hipertensão arterial \\
\hline $\mathbf{1 0}$ & $\begin{array}{l}\text { Fatores sócios demográficos que interferem na adesão do tratamento dos portadores de } \\
\text { hipertensão arterial }\end{array}$ \\
\hline $\mathbf{1 1}$ & Aspectos sócio-demográficos que influenciam na adesão ao tratamento anti-hipertensivo \\
\hline
\end{tabular}

Fonte: BVS.

\subsection{Fatores que influenciam à não adesão ao tratamento anti-hipertensivo}

Os estudos analisados acerca da temática apontaram múltiplos fatores que predispõem os indivíduos portadores de HA a não aderirem ao tratamento anti-hipertensivo, os fatores apontados pelos autores como causa da não adesão estão relacionados às razões ligadas à própria instituição assistencial e profissional de saúde, socioeconômicos e demográficos, aspectos psicossociais e culturais, apoio social e familiar, e ao tratamento terapêutico. A seguir alguns dos fatores enfatizados pelos autores.

Analisou-se um estudo que buscava identificar os motivos justificados pelos pacientes ao abandono do seguimento médico e terapêutico, os principais motivos relatados pelos participantes dessa pesquisa foram as razões ligadas à organização e estrutura do serviço, em decorrência de dificuldades, incompatibilidades e insatisfações com diferentes dimensões dos serviços prestados pela instituição, dentre as quais se destacaram o intervalo longo entre as consultas; dificuldade para agendar consulta; demora em ser atendido; horário de atendimento incompatível com o do trabalho ou com as ocupações diárias; mudança de médico responsável pela assistência e impossibilidade de acesso ao médico especialista. Nesse mesmo estudo, os participantes se mostraram descontentes e insatisfeitos com a assistência prestada pelos profissionais de saúde, sendo uma das causas apontada como fatores para o abandono do tratamento (DUARTE et al., 2010).

Diante dessas observações, percebeu-se que a estrutura e organização do serviço de saúde e a qualidade da assistência prestada pela equipe multiprofissional exerce uma forte influência quanto à motivação na adesão ao tratamento anti-hipertensivo. Dessa forma, observou-se que o acompanhamento ao paciente hipertenso tem sido realizado de forma inadequada, configurandose um fator determinante para a não adesão ao tratamento.

Em relação aos fatores ligados aos aspectos socioeconômicos e demográficos, identificaram- 
se algumas variáveis altamente relevantes, nos artigos analisados, como: Indivíduos do sexo masculino, idade avançada, baixa condição socioeconômica e de escolaridade estão associados à menor adesão ao tratamento. Porém outros estudos indicam a idade mais avançada como um facilitador do processo de adesão em relação aos mais jovens.

De acordo com Veras e Oliveira (2009), os indivíduos de idade mais avançada são mais propensos à adesão, visto que os jovens não se sentem vulneráveis à doença, enquanto os idosos são mais preocupados com a saúde e se apegam ao tratamento como alternativo de prolongamento da vida. Já, o fator escolaridade influi, diretamente, na assimilação das orientações acerca da patologia; portanto, quanto mais baixa a escolaridade, mais difícil se torna compreender o diagnóstico, a necessidade da mudança de hábitos e os esquemas posológicos. Vale ressaltar que a baixa renda interfere na aquisição de medicamento, quando este não está disponível na Unidade Básica de Saúde.

No Brasil, um dos primeiros estudos sobre os aspectos socioeconômicos e demográficos da população hipertensa evidenciou que a maior taxa de abandono ao tratamento da hipertensão ocorria entre homens, analfabetos e com idade entre vinte e quarenta anos. De forma similar, estudo mais recente, também observou que homens tendem a serem menos aderentes ao tratamento antihipertensivo (PIERIN et al., 2011).

Segundo o Ministério da Saúde (BRASIL, 2009), vários estudos comparativos, entre homens e mulheres, têm comprovado o fato de que os homens são mais vulneráveis às doenças, sobretudo às enfermidades graves e crônicas. A despeito da maior vulnerabilidade e das altas taxas de morbimortalidade, os homens não buscam, como as mulheres, os serviços de atenção básica. Tratamentos crônicos ou de longa duração têm, em geral, menor adesão, visto que os esquemas terapêuticos exigem um grande empenho do paciente que, em algumas circunstâncias, necessita modificar seus hábitos de vida para cumprir seu tratamento. Tal afirmação também é válida para ações de promoção e prevenção à saúde, que requerem, na maioria das vezes, mudanças comportamentais.

Em pesquisas qualitativas, apontaram-se várias razões, mas, de um modo geral, podemos agrupar as causas da baixa adesão em dois grupos principais de determinantes, que se estruturam como barreiras entre o homem e os serviços e ações de saúde, a saber: barreiras sócio culturais e barreiras institucionais. Grande parte da não adesão às medidas de atenção integral, por parte do homem, decorre das variáveis culturais (BRASIL, 2009).

Quanto ao apoio familiar, autores destacaram que indivíduos que compartilham experiências com seus cônjuges e deles recebem apoio, podem ter uma melhor participação no seguimento do tratamento, porém aqueles indivíduos que não conviviam com o cônjuge e residiam com filhos ou outras pessoas tinham uma adesão menor ao regime terapêutico. Isso pode ser atribuído à falta de estimulo que o hipertenso sofria para seguir o tratamento ou mesmo pela adequação que ele tinha que fazer para agradar os outros familiares (FIGUEIREDO; ASAKURA, 2010).

Percebe-se que a participação da família é fundamental para estimular o autocuidado dos indivíduos, os seus cônjuges apresentam-se como um ponto crucial facilitador para a resposta ao tratamento e à sua continuidade. 
Muitos pesquisadores também relacionam o uso da medicação como um dos problemas de não adesão, estudos encontrados na literatura atual citam como uma das principais causas a dificuldade de conseguir a medicação, pelo fato de não poderem se locomover até à Unidade Básica de Saúde, tendo em vista que a maioria é de idosos (BOSSAY et al., 2006).

Em outro estudo realizado com 353 hipertensos, evidenciou-se que os principais motivos que contribuem para que o paciente abandone o tratamento são: o alto custo dos medicamentos, a necessidade de tomá-lo várias vezes ao dia, a ocorrência de efeitos indesejáveis, o desconhecimento das complicações, a ausência de sintomatologia e o esquecimento. Soma-se a esses fatores o fato de que, quando o processo de conscientização é negligenciado, pode acontecer uso incorreto do medicamento e levar o paciente a não seguir as prescrições médicas de maneira satisfatória (SOARES et al., 2011).

No que se refere ao tratamento não farmacológico, Soares et al. (2011), evidenciaram, em sua pesquisa, que a maioria dos entrevistados não aderiu ao tratamento, principalmente por encontrarem dificuldades no cumprimento da dieta e da prática de atividade física. Esse fato pode estar associado ao entendimento de que as restrições da dieta, por exemplo, a diminuição da ingestão de sódio denota a ideia de castigo por estarem ligadas ao prazer em degustar alimentos, ou, ainda, associado à perda da liberdade de escolha.

Os aspectos associados à não adesão, aqui citados devem receber atenção especial pela complexidade dessa problemática, pois indicam a necessidade de políticas públicas voltadas para o favorecimento da adesão ao tratamento anti-hipertensivo.

\section{CONCLUSÃO}

O estudo realizado permitiu sintetizar a produção de conhecimento nacional acerca dos fatores que influenciam a não adesão ao tratamento da hipertensão. Durante a análise dos artigos, observou-se que a temática proposta é bastante atual, devido à dificuldade que muitos pacientes com hipertensão têm em adotar medidas de controle, o que despertou interesse de diversos profissionais da saúde, principalmente da enfermagem que presta uma assistência contínua a essa população.

Os resultados desta pesquisa evidenciaram que o ano de 2008 e 2010 concentrou o maior número de artigos, com $28 \%$ das publicações cada um. A abordagem mais frequente nos artigos selecionados foi a quantitativa, perfazendo um total de $73 \%$. A região geográfica onde se concentrou o maior número de pesquisa foi a sudeste, com $37 \%$ das publicações. Quanto ao local de realização da pesquisa, a Unidade Básica de Saúde se destacou com 91\%. É importante salientar que 73\% dos trabalhos selecionados foram realizados por enfermeiros e $55 \%$ foram encontrados em revistas especializadas de enfermagem.

Esse estudo possibilitou compreender que a adesão ao tratamento anti-hipertensivo é um processo complexo que envolve vários fatores que podem contribuir para a não adesão, tal fato se deve aos aspectos relacionados à condição dos serviços e profissionais de saúde, fatores socioeconômicos e demográficos, psicossociais e culturais e à participação da família no tratamento. 
Percebeu-se, ainda, que as pesquisas desenvolvidas sobre o assunto são de vital importância para a equipe multiprofissional da estratégia saúde da família, pois permitem a identificação dos problemas que envolvem essa clientela, assim facilita a elaboração de ações estratégicas que visam a aperfeiçoar a assistência para prevenir os problemas da não adesão ao tratamento. .

A luta contra a não adesão aos tratamentos da HA consiste em um grande desafio, tanto para o poder público como para os profissionais de saúde, pois depende da implementação de planos, projetos e programas multidisciplinares em todos os níveis de atendimento aos pacientes hipertensos para que as intervenções sejam mais eficazes e tenham a finalidade de evitar o abandono ao tratamento da HAS. Assim, para mudar essa realidade, são necessários mais investimentos financeiros na área da saúde, que visem à qualificação profissional, contratação de recursos humanos, construção de novas unidades de saúde, recursos materiais e, principalmente, no desenvolvimento de programas de saúde que atendam à realidade vivenciada por essa população.

Essas conclusões mostram que, mesmo sendo variados os motivos que levam aos hipertensos à não adesão ao tratamento, é importante compreender esses reais motivos retratados na literatura, pois, assim, é mais fácil criar projetos e ações de saúde pública voltados para esse agravo que traz tantas consequências maléficas à sociedade como um todo. Os autores do estudo sugerem uma reformulação nos programas assistenciais e o envolvimento de todos os profissionais para promover a adesão ao tratamento e a adoção de medidas saudáveis no estilo de vida de seus pacientes.

\section{REFERÊNCIAS}

BOSSAY, D. et al. Fatores associados à não-adesão ao tratamento anti-hipensivo. Ensaios e

Ciências, Campo Grande, v. 10, n. 3, 2006. Disponível em: <http://www.redalyc.org/articulo.oa?id=26012809008>. Acesso em: 12 dez. 2012.

BRASIL. Ministério da Saúde. Política Nacional de Atenção Integral à Saúde do Homem (princípio e diretrizes) Brasília, 2009. Disponível em: $<$ http://portal.saude.gov.br/portal/arquivos/pdf/politica nacional atencao integral.pdf >. Acesso em: 12 dez. 2012.

COSTA, J. S. D. et al. Prevalência de Hipertensão Arterial em Adultos e Fatores Associados: um Estudo de Base Populacional Urbana em Pelotas, Rio Grande do Sul, Brasil. Arq Bras Cardiol., v. 88, n. 1, 2007. Disponível em: <http://www.scielo.br/scielo.php?script=sci arttext\&pid=S0066782X2007000100010\&lng=en\&nrm=iso>. Acesso em: 12 set. 2012. http://dx.doi.org/10.1590/S0066-782X2007000100010.

DUARTE, M. T. C. et al. Motivos do abandono do seguimento médico no cuidado a portadores de hipertensão arterial: a perspectiva do sujeito. Ciênc. saúde coletiva, Rio de Janeiro, v. 15, n. 5, 2010. Disponível em: <http://www.scielo.br/scielo.php?script=sci arttext\&pid=S141381232010000500034\&lng=en\&nrm=iso $>$. Acesso em: 10 dez. 2012. 
FIGUEIREDO, N. N.; ASAKURA, L. Adesão ao tratamento anti-hipertensivo: dificuldades relatadas por indivíduos hipertensos. Acta Paul Enferm. São Paulo, v. 23, n. 6, 2010. Disponível em: $<$ http://www.scielo.br/pdf/ape/v23n6/11.pdf $>$. Acesso em: $10 \mathrm{dez} .2012$.

GUEDES, M. V. C. et al. Barreiras ao tratamento da hipertensão arterial. Rev. bras. enferm., Brasília, $\quad$ v. 64,6 n. $6,2011 . \quad$ Disponível em: $<$ http://www.scielo.br/scielo.php?script=sci arttext\&pid=S0034-

71672011000600008\&lng=en\&nrm=iso $>$. Acesso em: 02 set. 2012.

ISHITANI, L. H. et al. Desigualdade social e mortalidade precoce por doenças cardiovasculares no Brasil. Rev Saúde Pública, São Paulo, 2006. Disponível em: <http://www.scielo.br/pdf/rsp/v40n4/19.pdf>. Acesso em: 14 set. 2012

LOPES, G. G; FERREIRA, N. L.; MARIANO, P. N. Programa Saúde da família: assistência ao hipertenso. São Paulo, $2009 . \quad$ Disponível em: $<$ http://www.cpgls.ucg.br/ArquivosUpload/1/File/CPGLS/IV\%20MOSTRA/SADE/SAUDE/Programa \%20Sade\%20da\%20Famlia\%20 \%20Assistncia\%20a0\%20Hipertenso.pdf>. Acesso em: 09 dez. 2012.

MANFROI, A.; OLIVEIRA, F. A. Dificuldades de adesão ao tratamento na hipertensão arterial sistêmica: considerações a partir de um estudo qualitativo em uma unidade de Atenção Primária à Saúde. Rev Bras Med Fam e Com., Rio de Janeiro, v. 2, n. 7, 2006. Disponível em: $<$ http://www.rbmfc.org.br/rbmfc/article/view/52>. Acesso em: 12 set. 2012.

PIERIN, A. M. G. et al. Controle da hipertensão arterial e fatores associados na atenção primária em unidade básica de saúde localizadas na região oeste da cidade de São Paulo. Rev. Ciência Saúde, Rio de Janeiro, v. 16, supl. 1, 2011. Disponível em: $<$ http://www.scielo.br/scielo.php?script=sci arttext\&pid=S141381232011000700074\&lng=en\&nrm=iso $>$ Acesso em: 09 dez. 2012.

POMPEO, D. A.; ROSSI, L. A.; GALVÃO, C. M. Revisão integrativa: etapa inicial do processo de validação de diagnóstico de enfermagem. Acta. Paul. Enferm., São Paulo, v. 22, n. 4, 2009. Disponível em: <http://www.scielo.br/pdf/ape/v22n4/a14v22n4.pdf>. Acesso em: 05 out. 2012.

SANTA-HELENA, E. T.; NEMES, M. I. B.; ELUF NETO, J. Fatores associados à não-adesão ao tratamento com anti-hipertensivos em pessoas atendidas em unidades de saúde da família. Cad. Saúde Pública, Rio de Janeiro, 2010. Disponível em: $<$ http://www.scielo.br/scielo.php?script=sci arttext\&pid=S0102-

311X2010001200017\&lng=en\&nrm=iso > . Acesso em: 12 set. 2012.

SOARES, M. M. et al. Adesão do idoso ao tratamento da hipertensão arterial sistêmica: revisão integrativa. Cogitare Enferm., Curitiba, v. 17, n. 1, 2011. Disponível em: <http://ojs.c3sl.ufpr.br/ojs2/index.php/cogitare/article/viewFile/26389/17582>. Acesso em: $10 \mathrm{dez}$. 2012.

SOUZA, M. T.; SILVA, M. D.; CARVALHO, R. Revisão integrativa: o que é e como fazer. Einstein, São Paulo, v. 8, n. 1, 2010. Disponível em: <http://apps.einstein.br/revista/arquivos/PDF/1134Einsteinv8n1 p102-106 port.pdf>. Acesso em: 05 out. 2012. 
TEIXEIRA, E. As três metodologias: acadêmica da ciência e da pesquisa. 4. ed. UNAMA: BelémPA, 2002.

TOSCANO, C. M. As campanhas nacionais para detecção das doenças crônicas nãotransmissíveis: diabetes e hipertensão arterial. Ciênc. saúde coletiva, Rio de Janeiro, 2004. Disponível em: <http://www.scielosp.org/pdf/csc/v9n4/a10v9n4.pdf>. Acesso em: 12 set. 2012.

VERAS, R. F. S.; OLIVEIRA, J. S. Aspectos sócio-demográfico que influenciam na adesão ao tratamento anti-hipertensivo. Rev. Rene., Fortaleza, v. 10, n. 3, 2009. Disponível em: <http://www.revistarene.ufc.br/10.3/html/15.htm>. Acesso em: 09 dez. 2012. 